\title{
Effects of Platostoma palustre ethanolic extracts and commercial herbal tea on the cell viability of colorectal cancer cells
}

\author{
Yu-Hsing Lin 1, \#, Yun-Xuan Chang 2, \#, Chia-Chi Chen 2, \#, Hsiao-Yun Chen 2, \#, Ying-Ching Hung 2, \#, Tzu-Yun \\ $\mathrm{Chi}^{2}$, Chia-Yu Lin ${ }^{2}$, Guan-Hong Chen ${ }^{2}$, Ping-Min Huang ${ }^{2}$, Ya-Peng Wang ${ }^{2}$, Tsung-Han Wu ${ }^{2}$, Yen-Jung Lu ${ }^{2}$, \\ Chien-Chao Chiu ${ }^{2}$, Ching-Feng Chiu ${ }^{3}$, Hsuan-Wen Chiu ${ }^{4}$, Wei-Huang Tsai ${ }^{5}$ and Shao-Wen Hung 2, 6, ${ }^{*}$
}

\author{
${ }^{1}$ Bachelor Degree Program in Pet Healthcare, Yuanpei University of Medical Technology, Xiangshan, Hsinchu 300, Taiwan. \\ 2 Division of Animal Industry, Animal Technology Research Center, Agricultural Technology Research Institute, Xiangshan, \\ Hsinchu 300, Taiwan. \\ ${ }^{3}$ Graduate Institute of Metabolism and Obesity Sciences, College of Nutrition, Taipei Medical University, Taipei 110, Taiwan . \\ ${ }^{4}$ Department of Biotechnology and Bioindustry Sciences, College of Bioscience and Biotechnology, National Cheng Kung \\ University, Tainan 701, Taiwan. \\ ${ }_{5}^{5}$ Department of Science and Technology, Council of Agriculture, Executive Yuan, Taipei 100, Taiwan. \\ ${ }^{6}$ Department of Nursing, Yuanpei University of Medical Technology, Hsinchu 300, Taiwan. \\ \# Contributed equally to this work.
}

GSC Biological and Pharmaceutical Sciences, 2022, 18(02), 326-330

Publication history: Received on 21 January 2022; revised on 24 February 2022; accepted on 26 February 2022

Article DOI: https://doi.org/10.30574/gscbps.2022.18.2.0086

\begin{abstract}
Platostoma palustre jelly is a traditional food. Platostoma palustre has been used as folk medicine and is effective against heat-shock, hypertension and diabetes. Therefore, the aim of this study was to determine the effects of ethanolic extracts and commercial herbal tea of Platostoma palustre in inhibiting colorectal cancer cell viability. The ethanolic extracts of Platostoma palustre by using $90 \%$ ethanol for extraction. In this study, 2-fold serial dilution of $100 \mathrm{mg} / \mathrm{mL}$ Platostoma palustre extracts were applied. On other hand, the same dilution fold was also performed for $100 \%$ commercial herbal tea with Platostoma palustre. Additionally, CT-26 and HT-29 colorectal cancer cell lines were also used in this study. After co-culturing for 24 hours, the cell viability of CT-26 and HT-29 colorectal cancer cell lines were performed by using 3-(4, 5-Dimethylthiazol-2-yl)-2, 5-diphenyltetrazolium bromide (MTT) assay. According to these data, the 1.56-100 $\mathrm{mg} / \mathrm{mL}$ Platostoma palustre extracts possessed the significant inhibition effects of CT-26 colorectal cancer cell viability. The 3.13-100\% commercial herbal tea with Platostoma palustre possessed the significant inhibition effects of CT-26 colorectal cancer cell viability. The $6.25-100 \mathrm{mg} / \mathrm{mL}$ Platostoma palustre extracts possessed the significant inhibition effects of HT-29 colorectal cancer cell viability. The 25-100\% commercial herbal tea with Platostoma palustre possessed the significant inhibition effects of HT-29 colorectal cancer cell viability. However, the $0.39-3.13 \mathrm{mg} / \mathrm{mL}$ Platostoma palustre extracts possessed the significant promoting effects of HT-29 colorectal cancer cell viability. The 0.39-12.5\% commercial herbal tea with Platostoma palustre also possessed the significant promoting effects of HT-29 colorectal cancer cell viability. Comparison of CT-26 and HT-29 cell lines was on the cell viability after Platostoma palustre ethanolic extracts and commercial herbal tea treatments, CT-26 cell line was better sensitive than HT-29 cell line on the inhibition of cell viability after treatment of Platostoma palustre ethanolic extracts and the commercial herbal tea. Taken these results together, Platostoma palustre ethanolic extracts and commercial herbal tea may have a potential for inhibiting the growth of colorectal cancer cells.
\end{abstract}

Keywords: Cell viability; Colorectal cancer; Commercial herbal tea; Ethanolic extraction; In vitro; Platostoma palustre extracts

\footnotetext{
${ }^{*}$ Corresponding author: Shao-Wen Hung

Division of Animal Industry, Animal Technology Research Center, Agricultural Technology Research Institute, Xiangshan, Hsinchu 300, Taiwan.
}

Copyright ( 2022 Author(s) retain the copyright of this article. This article is published under the terms of the Creative Commons Attribution Liscense 4.0 


\section{Introduction}

Cancer is a major public health problem worldwide. The top 10 cancer types for estimated deaths in Taiwan in 2020 were in order as lung cancer, liver cancer, colorectal cancer, breast cancer (female), prostate cancer, oral cavity cancer, pancreatic cancer, stomach cancer, esophageal cancer, and ovarian cancer. Therefore, the research R\&D of novel antitumor drugs and other therapeutic strategies are urgently needed [1-3].

Platostoma palustre is an annual plant that is mainly distributed in tropical and subtropical regions, including Taiwan, Indonesia, Vietnam, southern China, and Burma [4-6]. The related food with Platostoma palustre as tea, herbal jelly, and sweet soup with herbal jelly are popular during the summer. Additionally, the heated herbal jelly with Platostoma palustre is admired by many Taiwanese in winter. Platostoma palustre has been used as folk medicine. Platostoma palustre has been verified that possessed many functional compounds such as polysaccharides (gum) with a unique aroma and texture, sterol compounds, stigmasterol, $\alpha$-sitosterol, tripterpene compounds, oleanolic acid, volatile compounds (caryophyllene oxide, $\alpha$-caryophyllene, eugenol, benzene acetaldehyde, and 2,3-butanedione etc), essential oil (n-hexadecanoic acid, linoleic acid, and linolenic acid), volatile oil (chavibetol, $n$-hexadecanoic acid, and $\alpha$-cadinol), and ursolic acid [4-8]. Therefore, theses functional compounds of Platostoma palustre have indicated that many biological effects is effective against and attenuating the metabolic syndrome, heat-shock, hypertension, diabetes, liver disease, muscle and/or joint pains, hyperglycemia, inflammation, oxidant activity, free radical scavenging effects, acute and chronic hepatitis, and caner growth [9-12].

The objective of this study was to evaluate the effects of Platostoma palustre extracts and commercial herbal tea on the cell viability of colorectal cancer cells. Therefore, we hypothesize that Platostoma palustre extracts via $90 \%$ ethanolic extraction and commercial herbal tea with Platostoma palustre can be effective in inhibiting CT-26 and HT-29 colorectal cancer cell viability in vitro. In addition, we also want to compare the tumoral inhibition abilities between CT-26 and HT-29 colorectal cancer cell lines after treating with Platostoma palustre extracts via 90\% ethanolic extraction and commercial herbal tea with Platostoma palustre.

\section{Material and methods}

\subsection{Cell Lines and Culture Condition}

CT-26 cells (ATCC ${ }^{\circledR}$ CRL-2638 ${ }^{\mathrm{TM}}$ ) and HT-29 (ATCC ${ }^{\circledR}$ HTB-38 ${ }^{\mathrm{TM}}$ ) were purchased from ATCC (Manassas, VA 20110). McCoy's 5a medium, RPMI-1640 medium, fetal bovine serum (FBS), and antibiotics (penicillin and streptomycin) were purchased from Sigma-Aldrich. CT-26 cells were cultured in RPMI-1640 medium and HT-29 cells were cultured in McCoy's 5a medium. Both McCoy's 5a medium and RPMI-1640 medium was supplemented with 10\% FBS and 1\% penicillin and streptomycin. The cells were incubated at $37^{\circ} \mathrm{C}$ with $5 \% \mathrm{CO}_{2}$. Cells were sub-cultured to replace flesh media per 2-3 days when they became confluent.

\subsection{Source of Dried Platostoma palustre and Commercial Herbal Tea}

The dried Platostoma palustre were collected from the traditional markets in Miaoli, Taiwan. The commercial herbal tea with Platostoma palustre were also purchased (Guanxi, Hsinchu, Taiwan).

\subsection{Chemical and Reagents}

Cell viability assay kit [3-(4, 5-Dimethylthiazol-2-yl)-2, 5-diphenyltetrazolium bromide; MTT] was purchased from $\operatorname{abcam}^{\circledR}$.

\subsection{0\% Ethanolic Extraction}

10-15 g samples of Platostoma palustre powder were soaked for $24 \mathrm{~h}$ at room temperature with $200 \mathrm{~mL}$ of $90 \%$ ethanol, followed by filtration through Whatman \#1 filter paper. The filtrates were then evaporated in vacuo to dryness and weighed to determine the yields.

\subsection{Cell Viability Assay}

CT-26 and HT-29 cells $\left(5 \times 10^{4} / \mathrm{mL}\right)$ were initially incubated for $24 \mathrm{~h}$ in a 96 well plate, respectively. The Platostoma palustre extracts and commercial herbal tea with Platostoma palustre were respectively diluted with culture media. Two-fold serial dilution of $100 \mathrm{mg} / \mathrm{mL}$ Platostoma palustre extracts were applied $(0,0.39,0.78,1.56,3.13,6.25,12.5$, 25, 50, and $100 \mathrm{mg} / \mathrm{mL}$ ). On other hand, the commercial herbal tea with Platostoma palustre was also performed 2-fold 
serial dilution $(0,0.39,0.78,1.56,3.13,6.25,12.5,25,50$, and $100 \%)$. After 24 h-experimental point, cell viability was detected by MTT cell viability assay kit. The reduced purple dye intensity of color was estimated by reading at optical density $570 \mathrm{~nm}$ in a spectrophotometer.

\subsection{Statistical Analysis}

The data were expressed as mean \pm SD. All comparisons were made by one-way ANOVA and all significant differences are reported at ${ }^{*} p<0.05,{ }^{* *} p<0.01,{ }^{* * *} p<0.001$, and ${ }^{* * * *} p<0.0001$

\section{Results}

\subsection{Suppression of Colorectal Cancer Cell Viability after Treatments of Platostoma palustre Ethanolic Extracts and Commercial Herbal Tea with Platostoma palustre}
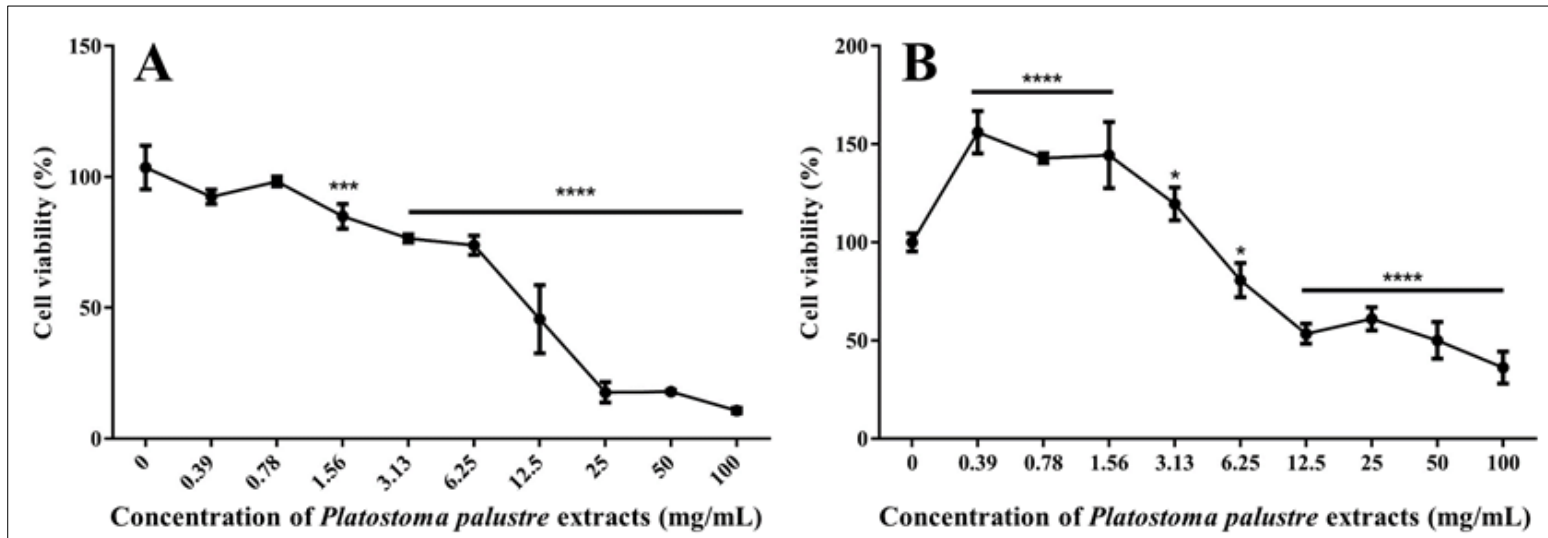

Figure 1 Inhibition cell viability abilities of colorectal cancer cell lines (CT-26 and HT-29) after Platostoma palustre extract treatment (24 hours). (A) CT-26 colorectal cancer cell line. (B) HT-29 colorectal cancer cell line. All data are expressed as mean \pm SD for three replicates. All significant differences compared to each other after Platostoma palustre extract treatment were reported at ${ }^{*} p<0.05,{ }^{* * *} p<0.001$, and ${ }^{* * * *} p<0.0001$

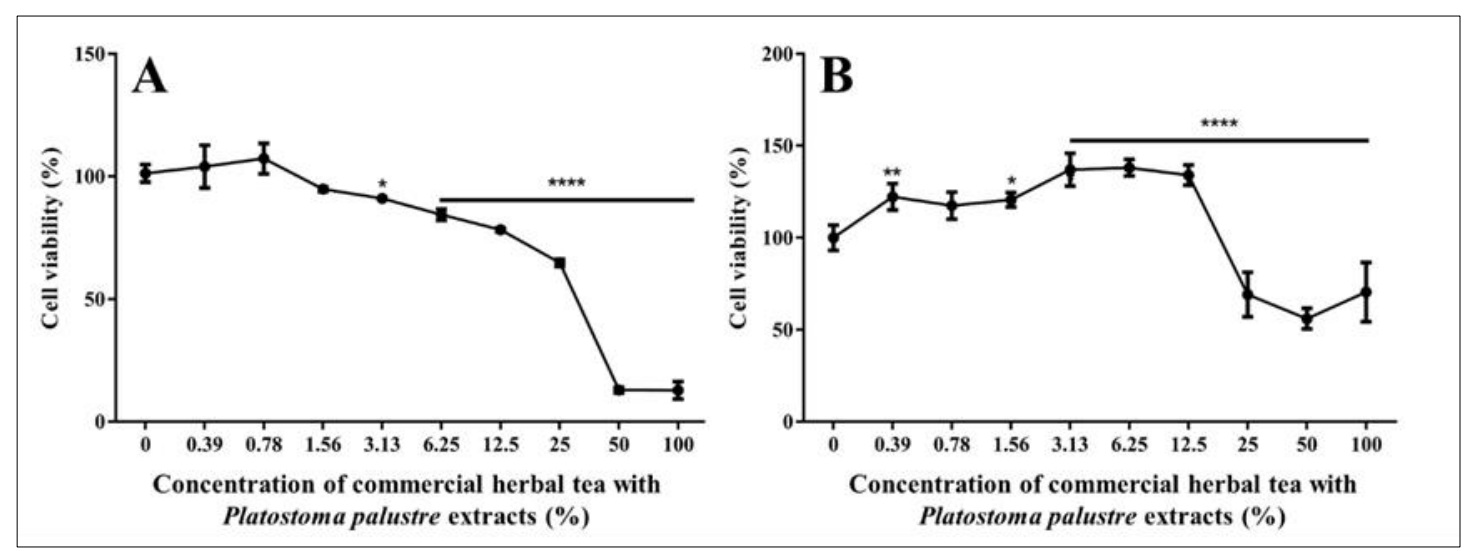

Figure 2 Inhibition cell viability abilities of colorectal cancer cell lines (CT-26 and HT-29) after the commercial herbal tea with Platostoma palustre treatment (24 hours). (A) CT-26 colorectal cancer cell line. (B) HT-29 colorectal cancer cell line. All data are expressed as mean \pm SD for three replicates. All significant differences compared to each other after the commercial herbal tea with Platostoma palustre treatment were reported at ${ }^{*} p<0.05,{ }^{* *} p<0.01$, and ${ }^{* * * *} p<0.0001$

The ethanolic extracts of Platostoma palustre by using 90\% ethanol for extraction. In this study, 2-fold serial dilution of $100 \mathrm{mg} / \mathrm{mL}$ Platostoma palustre extracts were applied. On other hand, the commercial herbal tea with Platostoma palustre was also performed 2-fold serial dilution. Additionally, CT-26 and HT-29 colorectal cancer cell lines were also used in this study. After co-culturing for 24 hours, the cell viability of CT-26 and HT-29 colorectal cancer cell lines were 
performed by using MTT assay. According to these data, the $1.56-100 \mathrm{mg} / \mathrm{mL}$ Platostoma palustre ethanolic extracts possessed the significant inhibition effects of CT-26 colorectal cancer cell viability (Fig. 1A). The 3.13-100\% commercial herbal tea with Platostoma palustre possessed the significant inhibition effects of CT-26 colorectal cancer cell viability (Fig. 1B). The $6.25-100 \mathrm{mg} / \mathrm{mL}$ Platostoma palustre extracts possessed the significant inhibition effects of HT-29 colorectal cancer cell viability (Fig. 2A). The 25-100\% commercial herbal tea with Platostoma palustre possessed the significant inhibition effects of HT-29 colorectal cancer cell viability (Fig. 2B). However, 0.39-3.13 mg/mL Platostoma palustre extracts possessed the significant promoting effects of HT-29 colorectal cancer cell viability (Fig. 2A). The 0.39$12.5 \%$ commercial herbal tea with Platostoma palustre also possessed the significant promoting effects of HT-29 colorectal cancer cell viability (Fig. 2B).

\section{Discussion}

The incidence and mortality of cancer are rapidly growing worldwide. The reasons are complex and both aging and growth of the population are related with the prevalence and distribution of the main risk factors for cancer. World Health Organization (WHO) in 2015 reported that cancer is the first or second leading cause of death in 91 of 172 countries. WHO lists cancer was one of top 10 threats to people health. Nearly, 10 million people die of cancer each year worldwide. Additionally, some reports presented that the global cancer cases will be increase to $60 \%$ in 2040 . There may be nearly 29.4 million new cases of cancer each year [1-3]. In Taiwan in 2020, the top 5 cancers among Taiwanese are lung cancer, liver cancer, colorectal cancer, female breast cancer, and prostate cancer. Cancer is also a threat to the lives of people in Taiwan. Therefore, the research $R \& D$ of novel anti-tumor drugs and other therapeutic strategies are urgently needed.

Previously, the effect of different ethanolic concentrations on antioxidant properties and cytoprotective activities of Platostoma palustre has been verified. The antioxidant activities of Mesona procumbens ethanolic extracts which displayed variable antioxidant levels. The 60\% Mesona procumbens ethanolic extracts exhibited higher antioxidant activities that possessed a protective capability for the biological membrane system to prevent and treat oxidative stress-related disorders [13-16]. In this study, 90\% Platostoma palustre ethanolic extracts were used. The bio-functional indexes and concentrations of $90 \%$ Platostoma palustre ethanolic extracts were chlorogenic acid $(0.30 \mathrm{mg} / \mathrm{mg} 90 \%$ Platostoma palustre ethanolic extracts), caffeic acid $(0.70 \mathrm{mg} / \mathrm{mg} 90 \%$ Platostoma palustre ethanolic extracts), rutin $(0.80 \mathrm{mg} / \mathrm{mg} 90 \%$ Platostoma palustre ethanolic extracts), astragaloside IV $(7.50 \mathrm{mg} / \mathrm{mg} 90 \%$ Platostoma palustre ethanolic extracts), and rosmarinic acid (15.90 mg/mg 90\% Platostoma palustre ethanolic extracts). Among of these bio-functional indexes of $90 \%$ Platostoma palustre ethanolic extracts, the contents of astragaloside IV and rosmarinic acid were higher than others. Many literatures have been demonstrated that astragaloside IV and rosmarinic acid possessed anti-tumor abilities. Therefore, we want to understand the effects of Platostoma palustre extracts and commercial herbal tea on the cell viability of colorectal cancer cells. In vitro study showed that comparison of CT-26 and HT-29 cell lines was on the cell viability after Platostoma palustre ethanolic extracts and commercial herbal tea treatment, CT-26 cell line was better sensitive than HT-29 cell line on the inhibition of cell viability after treatment of Platostoma palustre ethanolic extracts and commercial herbal tea.

\section{Conclusion}

Platostoma palustre has been used as a folk medicine and is effective against heat-shock, hypertension and diabetes. Therefore, the aim of this research was to determine the effects of ethanolic extracts and commercial herbal tea of Platostoma palustre in inhibiting colorectal cancer cell viability. Comparison of CT-26 and HT-29 cell lines was on the cell viability after Platostoma palustre ethanolic extracts and commercial herbal tea treatment, CT-26 cell line was better sensitive than HT-29 cell line on the inhibition of cell viability after treatment of Platostoma palustre ethanolic extracts and commercial herbal tea. Taken these results together, Platostoma palustre ethanolic extracts and commercial herbal tea may have a potential for inhibiting the growth of colorectal cancer cells. We hope that Platostoma palustre ethanolic extracts will be more deeply researched in the R\&D of new anti-colorectal cancer drug in the future.

\section{Compliance with ethical standards}

\section{Acknowledgments}

All authors thank the Council of Agriculture in Taiwan (Executive Yuan) [grant numbers 110 AS-14.3.2-ST-a2] for supporting this study. 


\section{Disclosure of conflict of interest}

The authors declare no conflict of interest.

\section{References}

[1] Bray F, Ferlay J, Soerjomataram I, Siegel RL, Torre LA, Jemal A. Global cancer statistics 2018: GLOBOCAN estimates of incidence and mortality worldwide for 36 cancers in 185 countries. CA. Cancer J. Clin. 2018; 68: 394424.

[2] Chen CC, Lin CY, Chi TY, Hung YC, Chen HY, Chen YH, Chiu CC, Huang PM, Wu TH, Lin YH, Lin JS, Chiu CF, Chiu HW, Tsai WH, Lin PH, Hsu SF, Hung SW. Common orthotopic establishment of four cancer-bearing mouse models. United J. Biomed. Eng. Sci. 2020; 1: 1-4.

[3] Siegel RL, Miller KD, Jemal A. Cancer Statistics. 2020. Ca. Cancer J. Clin. 2020; 70: 7-30.

[4] Hsieh Y, Lin SP, Wu L, Fang W, Hwang TS. Effects of Antrodia camphorata extracts on anti-oxidation, antimutagenesis and protection of DNA against hydroxyl radical damage. BMC Complement Altern. Med. 2015; 15: 237.

[5] Huang DJ, Chen HJ, Lin CD, Lin YH. Antioxidant and antiproliferative activities of water spinach (Ipomoea aquatica Forsk) constituents. Bot Bull Acad. Sin. 2005; 46: 99-106.

[6] Pervin M, Hasnat MA, Lee YM, Kim DH, Jo JE, Lim BO. Antioxidant activity and acetylcholinesterase inhibition of grape skin anthocyanin (GSA). Molecules. 2014; 19: 9403-9418.

[7] Pourmorad F, Hosseinimehr SJ, Shahabimajd N. Antioxidant activity, phenol and flavonoid contents of some selected Iranian medicinal plants. Afr. J. Biotechnol. 2006; 5: 1142-1145.

[8] Yen GC, Chen HY. Antioxidant activity of various tea extracts in relation to their antimutagenicity. J .Agric. Food. Chem. 1995; 43: 27-32.

[9] Widyaningsih TD, Adilaras P. Hepatoprotective effect of extract of black Cincau (Mesona palustris BL) on paracetamol-induced liver toxicity in rats. Adv. J. Food Sci. Technol. 2013; 5: 1390-1394.

[10] Ahmed MB, Khater MR. Evaluation of the protective potential of Ambrosia maritima extract on acetaminopheninduced liver damage. J. Ethnopharmacol. 2001; 75: 169-174.

[11] Bessems JG, Vermeulen NP. Acetaminophen (acetaminophen)-induced toxicity: molecular and biochemical mechanisms, analogues and protective approaches. Crit. Rev. Toxicol. 2001; 31: 55-138.

[12] Kumar A, Sanjiv S, Chandel S. Evaluation of hepatoprotective activity of Abelmoschus moschatus seed in paracetamol induced hepatotoxicity on rat. J. Pharmacy. 2012; 2: 43-50.

[13] Okawa M, Kinjo J, Nohara T, Ono M. DPPH (1, 1-Diphenyl-2-Picrylhydrazyl) radical scavenging activity of flavonoids obtained from some medicinal plants. Boil. Pharm. Bull. 2001; 24: 1202-1205.

[14] Widyaningsih T, Sukardiman D. Djoko AP, Win D. Immunomodulatory effects of the water extract of black Cincau (Mesona palustris BL) against interferon gamma expression, immunosurveillance activation and apoptosis on benzo (a) pyrene-induced fibrosarcoma carcinogenesis in mice. J. Technol. Food Ind. 2012; 23: 29-35.

[15] Yen GC, Hung CY. Effects of alkaline and heat treatment on antioxidative activity and total phenolic of extracts from Hsian-tsao (Mesona procumbens Hemsl.). Food Res. Int. 2001; 33: 487-492.

[16] Yen GC, Yeh CT, Chen YT. Protective effect of Mesona procumbens against tert-butyl hydroperoxide-induced acute hepatic damage in rats. J. Agric. Food Chem. 2004; 52: 4121-4127. 